\title{
Sex differences in associations between insulin resistance, heart rate variability and arterial stiffness in healthy women and men: a physiology study
}

\begin{tabular}{|r|l|}
\hline Journal: & Canadian Journal of Physiology and Pharmacology \\
\hline Manuscript ID & cjpp-2016-0122.R2 \\
\hline Manuscript Type: & Article \\
\hline Complete List of Authors: & $\begin{array}{r}\text { Rannelli, Luke; University of Calgary, Department of Internal Medicine } \\
\text { MacRae, Jennifer; University of Calgary, Faculty of Medicine, Alberta } \\
\text { Kidney Disease Network, Libin Cardiovascular Institute of Alberta } \\
\text { Mann, Michelle; University of Calgary, Faculty of Medicine, Libin } \\
\text { Cardiovascular Institute of Alberta } \\
\text { Ramesh, Sharanya; University of Calgary, Faculty of Medicine, Libin } \\
\text { Cardiovascular Institute of Alberta } \\
\text { Hemmelgarn, Brenda; University of Calgary, Faculty of Medicine, Alberta } \\
\text { Kidney Disease Network, Libin Cardiovascular Institute of Alberta } \\
\text { Rabi, Doreen; University of Calgary, Faculty of Medicine, Libin } \\
\text { Cardiovascular Institute of Alberta, Institute for Public Health } \\
\text { Sola, Dola; University of Calgary, Faculty of Medicine, Libin Cardiovascular } \\
\text { Institute of Alberta } \\
\text { Ahmed, Sofia; University of Calgary, Faculty of Medicine, Alberta Kidney } \\
\text { Disease Network, Libin Cardiovascular Institute of Alberta }\end{array}$ \\
\hline Keyword: & $\begin{array}{l}\text { Insulin Resistance, Cardiac Autonomic Tone, Women, Heart Rate } \\
\text { Variability, Arterial Stiffness }\end{array}$ \\
\hline
\end{tabular}

\section{SCHOLARONE"}

Manuscripts 
1 Sex differences in associations between insulin resistance, heart rate variability and

2 arterial stiffness in healthy women and men: a physiology study

3

4 Luke Anthony Rannelli MD MSc ${ }^{1}$, Jennifer M. MacRae MD MSc ${ }^{1,2,3}$, Michelle C. Mann

$5 \quad \mathrm{PhD}^{1,3}$, Sharanya Ramesh BSc ${ }^{1,3}$, Brenda R. Hemmelgarn MD PhD ${ }^{1,2,3,4}$, Doreen Rabi

$6 \mathrm{MD} \mathrm{MSc}^{1,3,4}$, Darlene Y. Sola $\mathrm{BScN}^{2,3}$, Sofia B. Ahmed MD MMSc ${ }^{1,2,3}$

$8{ }^{1}$ Faculty of Medicine, University of Calgary, Calgary, Alberta, Canada

$9 \quad{ }^{2}$ Alberta Kidney Disease Network

$10{ }^{3}$ Libin Cardiovascular Institute of Alberta

$11{ }^{4}$ Institute for Public Health, University of Calgary

16 Word Count (text only): 3170, Tables: 2, Figures: 2, References: 45

18 Corresponding author and person to whom reprint requests should be addressed:

19 Sofia B. Ahmed MD MMSc FRCPC

$20 \quad 1403-29^{\text {th }}$ St. NW, C210, Calgary, Alberta, Canada, T2N 2T9

21 Telephone: (403) 944-2745

22 Fax: (403) 944-2876

23 Email: sofia.ahmed@albertahealthservices.ca 


\section{Abstract}

35 Diabetes confers greater cardiovascular risk to women compared to men. Whether insulin

36 resistance-mediated risk extends to the healthy population is unknown. Measures of

37 insulin resistance (fasting insulin, homeostatic model assessment, hemoglobin A1c,

38 quantitative insulin sensitivity check index, glucose) were determined in 48 (56\% female)

39 healthy subjects. Heart-rate variability was calculated by spectral power analysis and

40 arterial stiffness was determined using noninvasive applanation tonometry. Both were

41 measured at baseline and in response to angiotensin II infusion. In women, there was a

42 non-statistically significant trend towards increasing insulin resistance being associated

43 with an overall unfavourable HRV response and increased arterial stiffness to the

44 stressor, while men demonstrated the opposite response. Significant differences in the

45 associations between insulin resistance and cardiovascular physiological profile exist

46 between healthy women and men. Further studies investigating the sex differences in the

47 pathophysiology of insulin resistance in cardiovascular disease are warranted

54 Key Words: insulin resistance, cardiac autonomic tone, human, women, heart rate

55 variability, arterial stiffness, cardiovascular, angiotensin II 


\section{Introduction}

57 Insulin resistance is a stronger cardiovascular risk in women compared to men (Oterdoom

58 et al. 2009). The risk factors for early cardiac autonomic impairment differ among

59 diabetic women and men (Nolan et al. 2009) and fasting insulin level is a stronger

60 determinant of arterial stiffness (Giltay et al. 1999) and cardiovascular outcomes

61 (Oterdoom et al. 2009; Sarwar et al. 2007) in women than in men.

62 Impaired cardiac autonomic nervous system activity and increased arterial resistance,

63 both known complications of insulin resistance, are each associated with greater

64 cardiovascular risk (Vinik and Ziegler 2007; Yki-Jarvinen et al. 2007). The impact of

65 insulin resistance on HRV, a measure of cardiac autonomic tone, differs by sex (Nolan et

66 al. 2009) though whether these findings extend to the healthy population is unclear.

67 Similarly, while individuals with impaired fasting glucose have higher stress measures of

68 arterial stiffness, (Vasu et al. 2015) it is unknown whether this effect is modified by sex.

69 We sought to determine the association between insulin resistance and HRV and arterial

70 stiffness in healthy premenopausal women and men.

72 Methods

73 This study was approved by the University of Calgary Conjoint Health Research Ethics

74 Board. Subjects provided informed written consent. All subjects were healthy

75 normotensive, non- diabetic non-smokers and taking no prescription medications

76 (including oral contraceptives). Subjects underwent a medical history, physical

77 examination, and laboratory screening. Women were studied mid-menstrual cycle and 
78 none were on the oral contraceptive. All subjects maintained a high-salt state $(>150$

$79 \mathrm{mmol} /$ day) for 3 days prior to the study to ensure maximum renin angiotensin system

80 suppression (Shoback et al. 1983). Compliance with the high-salt diet was verified by

81 measuring sodium excretion through a 24-h urine collection or a second morning void

82 spot urine (Kawasaki et al. 1993). Each study commenced at 0800h following an

83 overnight fast in a quiet, temperature-controlled room with subjects in a supine

84 position.Blood pressure was measured via an automated sphygmomanometer (Dinamap,

85 GE Healthcare, USA) every $15 \mathrm{~min}$. Subjects were infused intravenously with angiotensin

86 II (AngII) $(3 \mathrm{ng} / \mathrm{kg} / \mathrm{min} \times 30 \mathrm{~min} ; 6 \mathrm{ng} / \mathrm{kg} / \mathrm{min} \times 30 \mathrm{~min})$.

87 Ambulatory electrocardiogram data were collected continuously (SEER MC

88 recorder, GE Healthcare). HRV frequency measures were calculated according to

89 standard methods (MARS version 7, GE Healthcare). Frequency domain parameters were

90 derived using power spectral analysis, where a combination of sympathetic and vagal

91 autonomic nervous system (ANS) power was represented by the low-frequency (LF; ms ${ }^{2}$ )

92 band within the range of $0.04-0.15 \mathrm{~Hz}$, and vagal power was represented by the high-

93 frequency $\left(\mathrm{HF} ; \mathrm{ms}^{2}\right)$ band within the range of $0.15-0.40 \mathrm{~Hz}$, both of which were

94 expressed in normalized units (nu) to account for changes in total power. The LF:HF

95 ratio component was calculated by comparing crude LF and HF parameters, representing

96 total cardiosympathovagal balance. Impaired cardiac autonomic tone, a known

97 complication of insulin resistance, is associated with increased mortality (Vink et al.

98 2007). HRV, represents a balance between cardiac sympathetic (LF) and parasympathetic

99 tone (HF) (vagal tone), with greater variability in heart rate considered a marker of a

100 healthy autonomic nervous system (Karayannis et al. 2012). 
101 The bPWV and AIx were measured noninvasively with applanation tonometry (Millar

102 Instruments, Houston, TX) and commercially available acquisition and analysis software

103 (Version 8 SphygmoCor; AtCor Medical, Sydney, Australia) every 15 min, as previously

104 described (Laurent et al. 2006). Subjects were studied in the supine position using a

105 standard cuff placed on the right arm. Two readings were taken and recorded at each time

106 point by the same registered nurse and the mean was reported. The peripheral vascular

107 stiffness was represented by bPWV, while the systemic vascular stiffness was represented

108 by AIx. Aortic augmentation is a validated measure of central vascular stiffness and is

109 also associated with adverse cardiovascular outcomes (Blacher et al. 1998; Kelly et al.

110 2001; Livingstone et al. 2013; Oparil et al. 2006; Smulyan et al. 2003). Serum insulin

111 levels were determined by chemiluminescent immunoassay (Abbott Diagnostics, USA),

112 HbA1c levels by turbidometric immunoassay and serum glucose by hexokinase UV

113 colorimetric (both Roche Diagnostics; Germany). Homeostatic Model Assessment

114 Insulin Resistance (HOMA-IR) was calculated as [(Fasting Glucose (mmol/l))x(Fasting

$115 \operatorname{Insulin}(\mu \mathrm{mol} / 1))] / 22.5$ (Wallace et al. 2004) and Quantitative Insulin Sensitivity Check

116 Index (QUICKI) as 1/[log(Fasting Insulin $(\mu \mathrm{mol} / \mathrm{l}))+\log ($ Fasting Glucose(mg/dl) $)]($ Katz

117 et al. 2000).

118 The exploratory primary analysis examined the association between parameters of

119 insulin resistance (fasting insulin, HOMA-IR, HbA1c, QUICKI, glucose) and HRV and

120 arterial stiffness at baseline and in response to 60min AngII challenge, stratified by sex.

121 Baseline and response to AngII measures were compared using Student t-test.

122 Frequencies were compared by $\chi^{2}$ tests. A stepwise selection multivariate linear

123 regression was applied to determine the relative contributions of covariates (for HRV: 
124 sex, age, body mass index (BMI), MAP, heart rate, high-density lipoprotein (HDL), low-

125 density lipoprotein (LDL), serum 25-hydroxyvitamin D, serum estradiol (women),

126 testosterone (men), and where appropriate, baseline LF, HF, or LF:HF; for arterial

127 stiffness: sex, age, BMI, MAP, HDL, LDL, serum 25-hydroxyvitamin D, uric acid, and

128 where appropriate, baseline AIx and bPWV). All model assumptions were tested and

129 met. Analyses were performed using Stata (version 10.0, STATA Corp. College Station

130 Tx) with $\alpha=0.05$.

131

132 Results

133 The characteristics of the 48 healthy subjects (56\% female, $75 \%$ Caucasian) enrolled

134 have been published previously (Mann et al. 2012; Ramesh et al. 2015; Samimi et al.

135 2014) and are outlined in Table 1. All subjects had normal measures of insulin resistance

136 (Chen et al. 2005), baseline HRV (Nunan et al. 2010) and baseline arterial stiffness

137 (Laurent et al. 2016).

138 Neither women nor men demonstrated any association between any measure of

139 insulin resistance and measures of baseline HRV or measures of baseline arterial

140 stiffness. However, on univariate analysis, there was a non-significant trend towards an

141 increased LF response to AngII $(r=0.27, p=0.1 ; p=0.07$ on multivariate analysis $)$ and a

142 more blunted HF response $(r=-0.24, p=0.16 ; p=0.07$ on multivariate analysis $)$ with

143 increasing fasting insulin levels in women. This was reflected in a non-significant

144 increase in overall LF:HF ( $p=0.1$ vs baseline). When adjusted for covariates, this

145 increased overall cardiosympathovagal balance approached significance ( $p=0.05$ vs

146 baseline) (Figure 1). Men demonstrated the reverse association between insulin levels and 
147 the LF $(\mathrm{p}=0.02)$ (women vs men LF, $p=0.06)$ and HF $(p=0.009)$ (women vs men HF,

$148 \mathrm{p}=0.07$ ) responses to AngII (women vs men LF:HF response: $p=0.049$ ) when adjusted for

149 all other covariates (Figure 1).

150 Similarly, men showed a negative relationship between HOMA-IR and LF ( $p=0.05)$

151 response to Ang II challenge and a significantly positive association with HF ( $p=0.02)$.

152 While not statistically significant, an opposite relationship was observed in women with a

153 positive trend between HOMA-IR and the LF response to AngII $(p=0.1)$ on multivariate

154 analysis and a negative trend with the HF $(p=0.15)$. No associations were observed

155 between $\mathrm{HbA1c}$ and measures of HRV in response to AngII challenge in either sex.

156 In women, increasing insulin sensitivity as demonstrated by QUICKI measurements was

157 associated with a trend towards decreasing $\operatorname{LF}(p=0.1)$ and increasing $\mathrm{HF}(p=0.1)$

158 responses to AngII, while men showed the opposite association (LF, $p=0.016 ; p=0.003$ vs

159 female response; $\mathrm{HF}, p=0.013 ; p=0.002$ vs. female response) on multivariate analysis.

160 Women did not demonstrate any association between fasting glucose and any HRV

161 measure in response to AngII challenge. Men showed no association between glucose

162 and the change in LF in response to AngII but glucose was associated with the HF

163 response to AngII $(r=0.34, p=0.046)$ and this was statistically different from women

$164(p=0.018)$. Results of the heart rate variability data are summarized in Table 2.

165 Arterial Stiffness response to AngII

166 As anticipated, all subjects demonstrated a significant increase in measures of arterial

167 stiffness (Table 2), with no differences between the female and male responses to AngII

168 (AIx: $p=0.41 ; \mathrm{PWV}, p=0.29$, women vs men). 
169 In response to AngII, however, women demonstrated a positive association between

170 fasting insulin levels and the AIx response $(r=0.37, p=0.023)$, a response not observed in

171 men (women vs men response, $p=0.07$ ). Similarly, HOMA-IR was positively associated

172 with the AIx response to AngII after adjustment for covariates in women $(p=0.03)$, but

173 not men (women vs men response, $p=0.07$ ) (Figure 2). There were no associations

174 between any of the other measures of insulin resistance and the AIx response to AngII in

175 either sex. No association was observed between measures of insulin resistance and

176 bPWV in either sex. There was no statistical significant difference between women and

177 men in MAP after AngII infusion. In addition no association was observed between

178 measures of insulin resistance and delta MAP in women or men.

179

180 Discussion

181 Our findings were as follows: 1) in women, increasing insulin resistance was not

182 associated with a change in HRV in response to a physiologic stressor, whereas men

183 showed a decrease in LF, a marker of cardiosympathetic activity and an increase in HF, a

184 marker of protective cardiovagal input with increasing levels of insulin resistance; 2)

185 increasing insulin resistance was associated with a greater increase in stress-related

186 arterial stiffness in women but not men. Our results add to the mounting evidence that

187 important sex differences exist in the pathophysiology by which insulin resistance affects

188 cardiovascular risk

189 Why sex modifies the cardiovascular risk profile associated with insulin

190 resistance is not clear (Shaw et al. 2006). A meta-analysis of 37 prospective cohort

191 studies of type 2 diabetes and fatal coronary heart disease with a total of 447,064 patients

192 demonstrated that the relative risk for fatal coronary heart disease associated 
193 with diabetes was 50\% higher in women compared to men (Huxley et al. 2006).

194 Compared to healthy subjects, diabetes was associated with a four- to fivefold higher 195 coronary artery disease rate among women, but not men (Kalyani et al. 2014). However,

196 investigation of the impact of diabetes or insulin resistance on the pathophysiology

197 leading to CVD is extremely limited. Impaired glucose tolerance is more common among 198 women, whereas men are more likely to demonstrate impaired fasting glucose (Cowie et 199 al. 2006), though how this may play into poorer cardiovascular outcomes in women is not 200 clear. Impaired glucose tolerance enhances non-enzymatic glycation and cross-linking of 201 collagen (Brownlee et al. 1988), which may thus predispose women to greater arterial 202 stiffness, though this remains speculative.

203 Studies examining sex differences in diabetic patients and HRV suggest that 204 significant differences exist between the sexes in the onset and severity in the 205 development of autonomic nervous system dysfunction (Nolan et al. 2009; Sengstock et 206 al. 2005). Nolan et al. 2009 reported that impaired HRV in women was associated with 207 the age at which the diagnosis of diabetes was made, whereas in men the development of 208 HRV abnormalities was associated with the duration of diabetes (Nolan et al. 2009). 209 Previous studies have suggested that differences in compliance and treatment intensity of 210 glycemic control may explain the sexual discrepancy in favour of men (Kramer et al. 211 2012), but this is unlikely given that none of the healthy subjects in the present study 212 were on treatment.

213 Endogenous sex hormones appear to play a role in mediating risk in the setting of 214 insulin resistance, with elevated levels of testosterone having opposing effects in men and 215 women (Ding et al. 2006). In older women, higher free testosterone levels were 
216 associated with greater insulin resistance (Oh et al. 2002). A meta-analysis examining the

217 role of sex hormones in the risk of developing diabetes reported a higher risk of type 2

218 diabetes among women with increased testosterone levels, with the opposite association

219 in men (Ding et al. 2006). It was postulated that the elevated testosterone at the levels

220 increases adipose tissue in women and therefore may subsequently increase in insulin

221 resistance, however the exact mechanism remains unclear (Lovejoy et al. 1996).

222 Similarly, low levels of sex-hormone binding globulin were associated with development

223 of diabetes in women but not in men, though a nested case-control study reported that

224 low circulating levels of sex hormone-binding globulin were a strong predictor of the risk

225 of type 2 diabetes in both women and men (Ding et al. 2006).

226 The role of circulating estrogen levels as a risk for developing diabetes in women

227 and men is less clear. In a meta-analysis, estradiol levels were elevated among men and

228 postmenopausal women with diabetes compared with controls, however there was no

229 sex-dimorphism between the groups (Ding et al 2006). There was insufficient prospective

230 data or studies to determine any additional association between estrogen levels and

231 insulin resistance (Ding et al. 2006). In a study of post-menopausal women, physiological

232 doses of estradiol did not alter arterial stiffness or insulin sensitivity (Vehkavaara et al.

233 2000). However, interpretation of the effects of estrogens on insulin resistance and

234 markers of cardiovascular disease remains challenging due to differences in age of study

235 populations, timing of menopause, and the type and route of administration of

236 postmenopausal hormone therapy (Grodstein et al. 2003). Of note, endogenous sex

237 hormone levels were accounted for in our analysis. Furthermore, none of the women in 
238 the study were ingesting the oral contraceptive and all women were studied at the same 239 phase of the menstrual cycle.

240 Fasting insulin is a strong predictor of cardiovascular disease in women. A meta241 analysis suggested that elevated fasting insulin concentrations conferred a greater

242 cardiovascular risk in women (Sarwar et al. 2007). In a study of 6916 non-diabetic 243 participants, every doubling of fasting insulin in women increased the risk of a 244 cardiovascular event by $50 \%$ even after exclusion of those with impaired fasting glucose, 245 an association not observed in men (Oterdoom et al. 2009).

246 Both depressed HRV and increased arterial stiffness are validated markers of 247 cardiovascular risk. Increased HOMA-IR is an independent risk factor for HRV 248 abnormalities in a young, non-diabetic population (Pal et al. 2013). In a systematic 249 review, HRV was reduced in women with metabolic syndrome with insulin resistance 250 highlighted as the potential principal factor responsible, though findings in men were 251 inconsistent (Stuckey et al. 2014). The vasodilating effect of insulin is blunted in subjects 252 with insulin resistance (Tamminen et al. 2002), suggesting another possible mechanism 253 by which increased insulin resistance augments cardiovascular risk. With the exception of 254 one male subject with elevated fasting insulin levels, none of the subjects in our study 255 had evidence of the metabolic syndrome, which may explain the lack of association 256 observed between insulin resistance and baseline measures of HRV and arterial stiffness.

257 We speculate that in the earliest stages of insulin resistance only a stressor may uncover 258 abnormalities in cardiac autonomic and arterial tone as observed in our study.

259 Due to the cross-sectional nature of our study, we cannot demonstrate 260 directionality of the association nor comment on causality; it is possible that 
261 dysfunctional HRV or arterial stiffness caused an increase in insulin resistance in women,

262 although this would not explain why the same phenomenon did not occur in men. While

263 the study of healthy non-diabetic subjects limits the generalizability of the results, by

264 studying a normotensive, healthy population, we aimed to examine the impact of insulin

265 resistance and sex on $\mathrm{HRV}$ and measures of arterial stiffness while minimizing

266 confounding factors. Second, we attempted to minimize the effect of sample size and

267 intra-individual variability by utilizing a homogenous study group and careful pre-study

268 design. We ensured that all participants were ingesting similar amounts of salt to ensure

269 maximum RAS suppression, that no female participant was ingesting oral contraceptives,

270 and that all female subjects were studied during the same stage of the menstrual cycle,

271 during the follicular (low estrogen) stage to control for the effect of estrogen on blood

272 pressure and the RAS. In addition, all subjects were studied at the same time of the day,

273 while resting in the supine position in a warm, quiet room after an 8-h fast. We measured

274 insulin resistance using the HOMA model rather than a hyperinsulinemic-euglycemic

275 clamp. However, the HOMA model is a validated clinical and epidemiological tool

276 (Wallace et al. 2004). Furthermore, measures of insulin resistance were collected at

277 baseline and we were thus unable to account for any potential changes in insulin

278 sensitivity as a result of an Ang II infusion. However a study examining the effect of a

279 concurrent hyperinsulinemic clamp and Ang II infusion in healthy non-diabetic stubjects

280 did not report any change to overall insulin sensitivity (Jonk et al. 2010). In a study of

281 healthy men and women, it was reported that random error represented a limited part of

282 the between-subject variability; therefore observed differences between individuals

283 mostly reflect differences in the subjects' error-free value rather than random error (Pinna 
284 et al. 2007). Furthermore, exclusion of one male subject who had a significantly higher

285 baseline serum insulin level showed a similar non-significant trend association between

286 insulin resistance and changes in LF and HF. This subject's BMI of 32 may contribute to

287 a higher circulating insulin level, though other measures of insulin resistance including

288 hemoglobin A1c (5.4\%), fasting glucose (5.6) HOMA-IR (4.98) and QUICKI (0.30) were

289 within the normal non-diabetic range and consistent with other studies of non-diabetic

290 obese males (Chen et al. 2005). AIx assesses systemic arterial stiffness, whereas PWV is

291 a measure of the arterial stiffness between two recording sites (Kim and Braam, 2013).

292 PWV determined from central arteries such as the aorta, carotid or femoral arteries is

293 considered the "gold standard" instead of the carotid-radial method utilized in this study.

294 However, the use of more peripheral arteries provided a less invasive method to measure 295 arterial stiffness (Laurent et al. 2016, Kim et al. 2013). Measurement of arterial stiffness

296 through brachial-ankle PWV has been demonstrated to provide similar predictive

297 information as that determined from central arterial stiffness in young healthy adults,

298 (Sugawara et al. 2005, Tsuchikura et al. 2010) similar to the population in our study. In a

299 study of healthy males which compared central aortic pulse wave transient time measured

300 by MRI to arterial stiffness measured by brachial PWV, there was close correlation

301 between the two measurement techniques. (Rezai et al. 2013). Finally, measurement of

302 the augmentation index (AIx) measured centrally and peripherally at the brachial site has

303 been compared and demonstrated to have a close correlation (Rezai et al. 2011).

304 Conclusion 
305 The results of this study suggest that insulin resistance confers a sex-dependent

306 unfavourable cardiac ANS response to a physiologic stressor. Further studies

307 investigating the pathophysiology of insulin resistance in CVD in women are warranted.

309 Acknowledgements

310 Author Contributions: LAR researched data, wrote manuscript; JMM researched data,

311 contributed to discussion, reviewed/edited manuscript; MCM researched data,

312 reviewed/edited manuscript; SR researched data, reviewed/edited manuscript; BRH

313 contributed to discussion, reviewed/edited manuscript; DR contributed to discussion,

314 reviewed/edited manuscript; DYS researched data, reviewed/edited manuscript; SBA

315 researched data, contributed to discussion, reviewed/edited manuscript). LAR and SBA

316 take responsibility for the contents of the article. This study was presented in abstract

317 form at the Canadian Society of Nephrology Annual Meeting in Montreal, Canada in

318 April 2015.

319 This study was funded by an Establishment Grant from Alberta Innovates - Health

320 Solutions. MCM is supported by the Canadian Institutes of Health Research. SR is

321 supported by the Roy and Vi Baay Chair Doctoral Scholarship. DR, BRH and SBA are

322 supported by Alberta Innovates - Health Solutions. JMM, BRH, DR and SBA are

323 supported by a joint initiative between Alberta Health and Wellness and the Universities

324 of Alberta and Calgary. Funding sources had no role in the design, conduct, or reporting

325 of this study.

326 The authors declare they have no competing interests. 
Blacher, J., Pannier, B., Guerin, A.P., Marchais, S.J., Safar, M.E., and London, G.M. 1998. Carotid arterial stiffness as a predictor of cardiovascular and all-cause mortality in end-stage renal disease. Hypertension, 32(3): 570-574.

331 Brownlee, M., Cerami, A., and Vlassara, H. 1988. Advanced glycosylation end products in tissue and the biochemical basis of diabetic complications. N. Engl. J. Med. 318(20): 1315-1321.

334 Chen, H., Sullivan, G., and Quon, M.J. 2005. Assessing the predictive accuracy of 335 QUICKI as a surrogate index for insulin sensitivity using a calibration model. Diabetes, 336 54(7): 1914-1925.

337 Cowie, C.C., Rust, K.F., Byrd-Holt, D.D., Eberhardt, M.S., Flegal, K.M., Engelgau, 338 M.M., Saydah, S.H., Williams, D.E., Geiss, L.S., and Gregg, E.W. 2006. Prevalence of diabetes and impaired fasting glucose in adults in the U.S. population: National Health And Nutrition Examination Survey 1999-2002. Diabetes Care, 29(6): 1263-1268.

341 Ding, E.L., Song, Y., Malik, V.S., and Liu, S. 2006. Sex differences of endogenous sex hormones and risk of type 2 diabetes: a systematic review and meta-analysis. JAMA 295(11): 1288-1299.

344 Giltay, E.J., Lambert, J., Elbers, J.M., Gooren, L.J., Asscheman, H., and Stehouwer, C.D. 345 1999. Arterial compliance and distensibility are modulated by body composition in both 346 men and women but by insulin sensitivity only in women. Diabetologia, 42(2): 214-221.

347 Grodstein, F., Clarkson, T.B., and Manson, J.E. 2003. Understanding the divergent data 348 on postmenopausal hormone therapy. N. Engl. J. Med. 348(7): 645-650.

349 Huxley, R., Barzi, F., and Woodward, M. 2006. Excess risk of fatal coronary heart 350 disease associated with diabetes in men and women: meta-analysis of 37 prospective 351 cohort studies. BMJ 332(7533): 73-78.

352 Jonk, A.M., Houben, A.J., Schaper, N.C., de Leeuw, P.W., Serne, E.H., Smulders, Y.M., 353 and Stehouwer, C.D. 2010. Angiotensin II enhances insulin-stimulated whole-body 354 glucose disposal but impairs insulin-induced capillary recruitment in healthy volunteers. J. 355 Clin. Endocrinol. Metab. 95(8): 3901-3908.

356 Kalyani, R.R., Lazo, M., Ouyang, P., Turkbey, E., Chevalier, K., Brancati, F., Becker, D., 357 and Vaidya, D. 2014. Sex differences in diabetes and risk of incident coronary artery

358 disease in healthy young and middle-aged adults. Diabetes Care, 37(3): 830-838. 
359 Karayannis, G., Giamouzis, G., Cokkinos, D.V., Skoularigis, J., and Triposkiadis, F.

360 2012. Diabetic cardiovascular autonomic neuropathy: Clinical implications. Expert

361 Review of Cardiovascular Therapy, 10(6): 747-765.

362 Katz, A., Nambi, S.S., Mather, K., Baron, A.D., Follmann, D.A., Sullivan, G., and Quon, M.J. 2000. Quantitative insulin sensitivity check index: A simple, accurate method for assessing insulin sensitivity in humans. J. Clin. Endocrinol. Metab. 85(7): 2402-2410.

365 Kawasaki, T., Itoh, K., Uezono, K., and Sasaki, H. 1993. A simple method for estimating $24 \mathrm{~h}$ urinary sodium and potassium excretion from second morning voiding urine specimen in adults. Clinical and Experimental Pharmacology and Physiology, 20(1): 7-14.

368 Kelly, R.P., Millasseau, S.C., Ritter, J.M., and Chowienczyk, P.J. 2001. Vasoactive drugs influence aortic augmentation index independently of pulse-wave velocity in healthy men. Hypertension 37(6): 1429-1433.

371 Kim, D.H. and Braam, B. 2013. Assessment of arterial stiffness using applanation

372 tonometry. Can. J. Physiol. Pharmacol. 91(12): 999-1008.

373 Kramer, H.U., Raum, E., Ruter, G., Schottker, B., Rothenbacher, D., Rosemann, T., 374 Szecsenyi, J., and Brenner, H. 2012. Gender disparities in diabetes and coronary heart 375 disease medication among patients with type 2 diabetes: results from the DIANA study.

376 Cardiovasc. Diabetol. 11: 88-2840-11-88.

377 Laurent, S., Cockcroft, J., Van Bortel, L., Boutouyrie, P., Giannattasio, C., Hayoz, D., 378 Pannier, B., Vlachopoulos, C., Wilkinson, I., Struijker-Boudier, H., and European 379 Network for Non-invasive Investigation of Large Arteries. 2006. Expert consensus 380 document on arterial stiffness: methodological issues and clinical applications. Eur. Heart 381 J. 27(21): 2588-2605.

382 Laurent, S., Marais, L., and Boutouyrie, P. 2016. The Noninvasive Assessment of 383 Vascular Aging. Can. J. Cardiol. 32(5): 669-679.

384 Livingstone, K.M., Lovegrove, J.A., Cockcroft, J.R., Elwood, P.C., Pickering, J.E., and 385 Givens, D.I. 2013. Does dairy food intake predict arterial stiffness and blood pressure in 386 men?: Evidence from the Caerphilly Prospective Study. Hypertension, 61(1): 42-47.

387 Lovejoy, J.C., Bray, G.A., Bourgeois, M.O., Macchiavelli, R., Rood, J.C., Greeson, C., 388 and Partington, C. 1996. Exogenous androgens influence body composition and regional 389 body fat distribution in obese postmenopausal women--a clinical research center study. J. 390 Clin. Endocrinol. Metab. 81(6): 2198-2203.

391 Mann, M.C., Exner, D.V., Hemmelgarn, B.R., Turin, T.C., Sola, D.Y., and Ahmed, S.B. 392 2012. Impact of gender on the cardiac autonomic response to angiotensin II in healthy

393 humans. J. Appl. Physiol. 112(6): 1001-1007. 
394 Nolan, R.P., Barry-Bianchi, S.M., Mechetiuc, A.E., and Chen, M.H. 2009. Sex-based

395

396

397

398

399

400

401

402

403

404

405

406

407

408

409

410

411

412

413

414

415

416

417

418

419

420

421

422

423

424

425

426

427

428

429 differences in the association between duration of type 2 diabetes and heart rate variability. Diabetes and Vascular Disease Research, 6(4): 276-282.

Nunan, D., Sandercock, G.R., and Brodie, D.A. 2010. A quantitative systematic review of normal values for short-term heart rate variability in healthy adults. Pacing Clin. Electrophysiol. 33(11): 1407-1417.

Oh, J.Y., Barrett-Connor, E., Wedick, N.M., Wingard, D.L., and Rancho Bernardo Study. 2002. Endogenous sex hormones and the development of type 2 diabetes in older men and women: the Rancho Bernardo study. Diabetes Care, 25(1): 55-60.

Oparil, S. and Izzo, J.L.,Jr. 2006. Pulsology rediscovered: commentary on the Conduit Artery Function Evaluation (CAFE) study. Circulation 113(9): 1162-1163.

Oterdoom, L.H., de Vries, A.P., Gansevoort, R.T., de Jong, P.E., Gans, R.O., and Bakker, S.J. 2009. Fasting insulin is a stronger cardiovascular risk factor in women than in men. Atherosclerosis, 203(2): 640-646.

Pal, G.K., Adithan, C., Ananthanarayanan, P.H., Pal, P., Nanda, N., Thiyagarajan, D., Syamsunderkiran, A.N., Lalitha, V., and Dutta, T.K. 2013. Association of sympathovagal imbalance with cardiovascular risks in young prehypertensives. Am. J. Cardiol. 112(11): 1757-1762.

Pinna, G.D., Maestri, R., Torunski, A., Danilowicz-Szymanowicz, L., Szwoch, M., La Rovere, M.T., and Raczak, G. 2007. Heart rate variability measures: a fresh look at reliability. Clin. Sci. (Lond) 113(3): 131-140.

Ramesh, S., Wilton, S.B., Holroyd-Leduc, J.M., Turin, T.C., Sola, D.Y., and Ahmed, S.B. 2015. Testosterone is associated with the cardiovascular autonomic response to a stressor in healthy men. Clin. Exp. Hypertens. 37(3): 184-191.

Rezai, M.R., Cowan, B.R., Sherratt, N., Finn, J.D., Wu, F.C., and Cruickshank, J.K. 2013. A magnetic resonance perspective of the pulse wave transit time by the Arteriograph device and potential for improving aortic length estimation for central pulse wave velocity. Blood Press. Monit. 18(2): 111-118.

Rezai, M.R., Goudot, G., Winters, C., Finn, J.D., Wu, F.C., and Cruickshank, J.K. 2011. Calibration mode influences central blood pressure differences between SphygmoCor and two newer devices, the Arteriograph and Omron HEM-9000. Hypertens. Res. 34(9): 1046-1051.

Samimi, A., Ramesh, S., Turin, T.C., MacRae, J.M., Sarna, M.A., Reimer, R.A., Hemmelgarn, B.R., Sola, D.Y., and Ahmed, S.B. 2014. Serum uric acid level, blood pressure, and vascular angiotensin II responsiveness in healthy men and women. Physiol. Rep. 2(12): 10.14814/phy2.12235. Print 2014 Dec 1. 
430 Sarwar N, Sattar N, Gudnason V, Danesh J. 2007. Circulating concentrations of insulin 431 markers and coronary heart disease: a quantitative review of 19 Western prospective 432 studies. European Heart Journal, 28(20): 2491-2497.

433 Sengstock, D.M., Vaitkevicius, P.V., and Supiano, M.A. 2005. Arterial stiffness is related 434 to insulin resistance in nondiabetic hypertensive older adults. J. Clin. Endocrinol. Metab. 435 90(5): 2823-2827.

436 Shaw, L.J., Bairey Merz, C.N., Pepine, C.J., Reis, S.E., Bittner, V., Kelsey, S.F., Olson, 437 M., Johnson, B.D., Mankad, S., Sharaf, B.L., Rogers, W.J., Wessel, T.R., Arant, C.B., 438 Pohost, G.M., Lerman, A., Quyyumi, A.A., Sopko, G., and WISE Investigators. 2006. 439 Insights from the NHLBI-Sponsored Women's Ischemia Syndrome Evaluation (WISE) 440 Study: Part I: gender differences in traditional and novel risk factors, symptom evaluation, 441 and gender-optimized diagnostic strategies. J. Am. Coll. Cardiol. 47(3 Suppl): S4-S20.

442 Shoback, D.M., Williams, G.H., Swartz, S.L., Davies, R.O., and Hollenberg, N.K. 1983. 443 Time course and effect of sodium intake on vascular and hormonal responses to enalapril 444 (MK 421) in normal subjects. J. Cardiovasc. Pharmacol. 5(6): 1010-1018.

445 Smulyan, H., Siddiqui, D.S., Carlson, R.J., London, G.M., and Safar, M.E. 2003. Clinical 446 utility of aortic pulses and pressures calculated from applanated radial-artery pulses.

447 Hypertension 42(2): 150-155.

448 Sugawara, J., Hayashi, K., Yokoi, T., Cortez-Cooper, M.Y., DeVan, A.E., Anton, M.A., 449 and Tanaka, H. 2005. Brachial-ankle pulse wave velocity: an index of central arterial 450 stiffness? J. Hum. Hypertens. 19(5): 401-406.

451 Tamminen, M., Westerbacka, J., Vehkavaara, S., and Yki-Jarvinen, H. 2002. Insulin452 induced decreases in aortic wave reflection and central systolic pressure are impaired in 453 type 2 diabetes. Diabetes Care, 25(12): 2314-2319.

454 Tsuchikura, S., Shoji, T., Kimoto, E., Shinohara, K., Hatsuda, S., Koyama, H., Emoto, M., 455 and Nishizawa, Y. 2010. Brachial-ankle pulse wave velocity as an index of central 456 arterial stiffness. J. Atheroscler. Thromb. 17(6): 658-665.

457 Vasu, S., Morgan, T.M., Kitzman, D.W., Bertoni, A., Stacey, R.B., Hamilton, C., Chiles, 458 C., Thohan, V., and Hundley, W.G. 2015. Abnormal stress-related measures of arterial 459 stiffness in middle-aged and elderly men and women with impaired fasting glucose at 460 risk for a first episode of symptomatic heart failure. J. Am. Heart Assoc. 4(1): e000991.

461 Vehkavaara, S., Westerbacka, J., Hakala-Ala-Pietila, T., Virkamaki, A., Hovatta, O., and 462 Yki-Jarvinen, H. 2000. Effect of estrogen replacement therapy on insulin sensitivity of 463 glucose metabolism and preresistance and resistance vessel function in healthy 464 postmenopausal women. J. Clin. Endocrinol. Metab. 85(12): 4663-4670. 
465 Vinik, A.I. and Ziegler, D. 2007. Diabetic cardiovascular autonomic neuropathy.

466 Circulation, 115(3): 387-397.

467 Wallace, T.M., Levy, J.C., and Matthews, D.R. 2004. Use and abuse of HOMA modeling. 468 Diabetes Care, 27(6): 1487-1495.

469 Yki-Jarvinen, H. and Westerbacka, J. 2007. Insulin resistance, arterial stiffness and wave 470 reflection. Adv. Cardiol. 44: 252-260.

471

472

473

474

475

476

477

478

479

480

481

482

483

484

485

486

487

488

489 
490 FIGURE 1. Heart rate variability response to Angll as a function of fasting insulin 491 level, by sex.

492 PANEL A - Low Frequency verses Insulin Level * $p=0.07$ vs. Response to 493 Women

494 PANEL B - High Frequency verse Insulin Level * $p=0.06$ vs. Response to 495 Women

496 PANEL C - Low Frequency:High Frequency Ration verses Insulin Level * $p$ $497<0.05$ vs. Response to Women

498

499 FIGURE 2. Arterial stiffness response to Angll challenge as a function of HOMA$500 \quad \mathrm{IR}$, by sex.

$501 * p=0.07$ vs Female Response

502 
Table 1. Baseline Characteristic: Baseline clinical and laboratory characteristics of women versus female subjects.

\begin{tabular}{|c|c|c|}
\hline Characteristic & Women $(n=27)$ & $\operatorname{Men}(n=21)$ \\
\hline Age (years) & $33 \pm 2$ & $40 \pm 3$ \\
\hline Caucasian (\%) & $71 \%$ & $81 \%$ \\
\hline $\mathrm{BMI}\left(\mathrm{Kg} / \mathrm{m}^{2}\right)$ & $24 \pm 0.7$ & $26 \pm 1$ \\
\hline $\begin{array}{l}\text { Mean Arterial Pressure } \\
(\mathrm{mmHg})\end{array}$ & $75 \pm 4$ & $88 \pm 3^{*}$ \\
\hline $\begin{array}{l}\text { Systolic Blood Pressure } \\
(\mathrm{mmHg})\end{array}$ & $105 \pm 4$ & $121 \pm 4^{*}$ \\
\hline $\begin{array}{l}\text { Diastolic Blood Pressure } \\
(\mathrm{mmHg})\end{array}$ & $62 \pm 3$ & $72 \pm 3 *$ \\
\hline Testosterone (nmol/1) & $1.5 \pm 0.1$ & $17.3 \pm 5^{*}$ \\
\hline Estradiol (pmol/l) & $402 \pm 65$ & $100 \pm 35^{*}$ \\
\hline Progesterone (nmol/l) & $8.9 \pm 3$ & $1.7 \pm 0.7^{*}$ \\
\hline Triglycerides (mmol/1) & $0.66 \pm 0.05$ & $0.96 \pm 0.1^{*}$ \\
\hline LDL (mmol/1) & $2.1 \pm 0.1$ & $2.47 \pm 0.13$ \\
\hline HDL (mmol/l) & $1.55 \pm 0.06$ & $1.23 \pm 0.06^{*}$ \\
\hline Fasting Glucose (mmol/l) & $4.5 \pm 0.1$ & $4.7 \pm 0.1$ \\
\hline Insulin (pmol/1) & $39 \pm 3$ & $43 \pm 6$ \\
\hline $\mathrm{HbAlc} \%$ (mmol/mol) & $5.5 \pm 0.05(37)$ & $5.5 \pm 0.1(37)$ \\
\hline HOMA-IR & $1.15 \pm 0.1$ & $1.33 \pm 0.2$ \\
\hline QUICKI & $0.38 \pm 0.05$ & $0.38 \pm 0.01$ \\
\hline
\end{tabular}

All data are expressed as mean \pm SE unless otherwise indicated.

Abbreviations: BMI, body mass index; LDL, Low-Density Lipoprotein; HDL, HighDensity Lipoprotein; HbA1C, Hemoglobin A1c; HOMA-IR, Homeostatic Model Assessment of Insulin Resistance; QUICKI, Quantitative Insulin Sensitivity Check Index $* \mathrm{P}<0.05$ vs women 
Table 2. Heart rate variability, arterial stiffness and blood pressure at baseline and in response to Angiotensin II infusion challenge in women and men.

\begin{tabular}{|c|c|c|c|c|}
\hline \multirow[b]{2}{*}{$\begin{array}{l}\text { Heart-Rate } \\
\text { Variability }\end{array}$} & \multicolumn{2}{|c|}{ Women $(n=27)$} & \multicolumn{2}{|c|}{ Male $(n=21)$} \\
\hline & Baseline & $\begin{array}{c}\text { Response to } \\
\text { Ang II } \\
\text { Challenge }\end{array}$ & Baseline & $\begin{array}{c}\text { Response to } \\
\text { Ang II } \\
\text { Challenge }\end{array}$ \\
\hline $\mathrm{LF}\left(\ln \mathrm{ms}^{2}\right)$ & $6.84 \pm 0.21$ & $6.89 \pm 0.19$ & $7.01 \pm 0.16$ & $7.15 \pm 0.15$ \\
\hline $\mathrm{HF}\left(\ln m s^{2}\right)$ & $6.33 \pm 0.21$ & $6.29 \pm 0.24$ & $5.86 \pm 0.24$ & $5.88 \pm 0.22$ \\
\hline $\mathrm{LF}(\mathrm{nu})$ & $59.57 \pm 2.58$ & $61.69 \pm 2.05$ & $72.38 \pm 2.76$ & $75.35 \pm 2.08^{*}$ \\
\hline HF (nu) & $37.29 \pm 2.69$ & $35.34 \pm 2.09$ & $25.61 \pm 2.57$ & $22.96 \pm 1.97^{*}$ \\
\hline LF:HF (nu) & $1.35 \pm 0.08$ & $1.39 \pm 0.08$ & $1.87 \pm 0.15$ & $1.96 \pm 0.13^{*}$ \\
\hline $\begin{array}{l}\text { Arterial } \\
\text { Stiffness }\end{array}$ & & 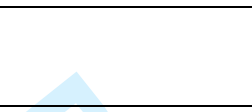 & & \\
\hline AIx & $8.63 \pm 2.57$ & $17.35 \pm 2.47$ & $2.55 \pm 3.67$ & $14.29 \pm 2.72$ \\
\hline PWV & $7.22 \pm 0.2$ & $8.74 \pm 0.4$ & $7.87 \pm 0.2$ & $9.39 \pm 0.3$ \\
\hline $\begin{array}{l}\text { Blood } \\
\text { Pressure }\end{array}$ & & & & \\
\hline $\begin{array}{l}\text { Systolic } \\
(\mathrm{mmHg})\end{array}$ & $106 \pm 4$ & $130 \pm 3$ & $119 \pm 3$ & $140 \pm 6$ \\
\hline $\begin{array}{l}\text { Diastolic } \\
\text { (mmHg) }\end{array}$ & $62 \pm 3$ & $80 \pm 2$ & $71 \pm 2$ & $98 \pm 2$ \\
\hline
\end{tabular}

All data are expressed as mean $\pm \mathrm{SE}$ unless otherwise indicated. $\Delta$ values in parentheses indicate mean change from baseline. $* \mathrm{p}<0.05$ versus responses to women.

Abbreviations: Ang, angiotensin; LF, low-frequency; HF, high-frequency; LF:HF, highfrequency-low-frequency ratio; AIx, aortic augmentation index 

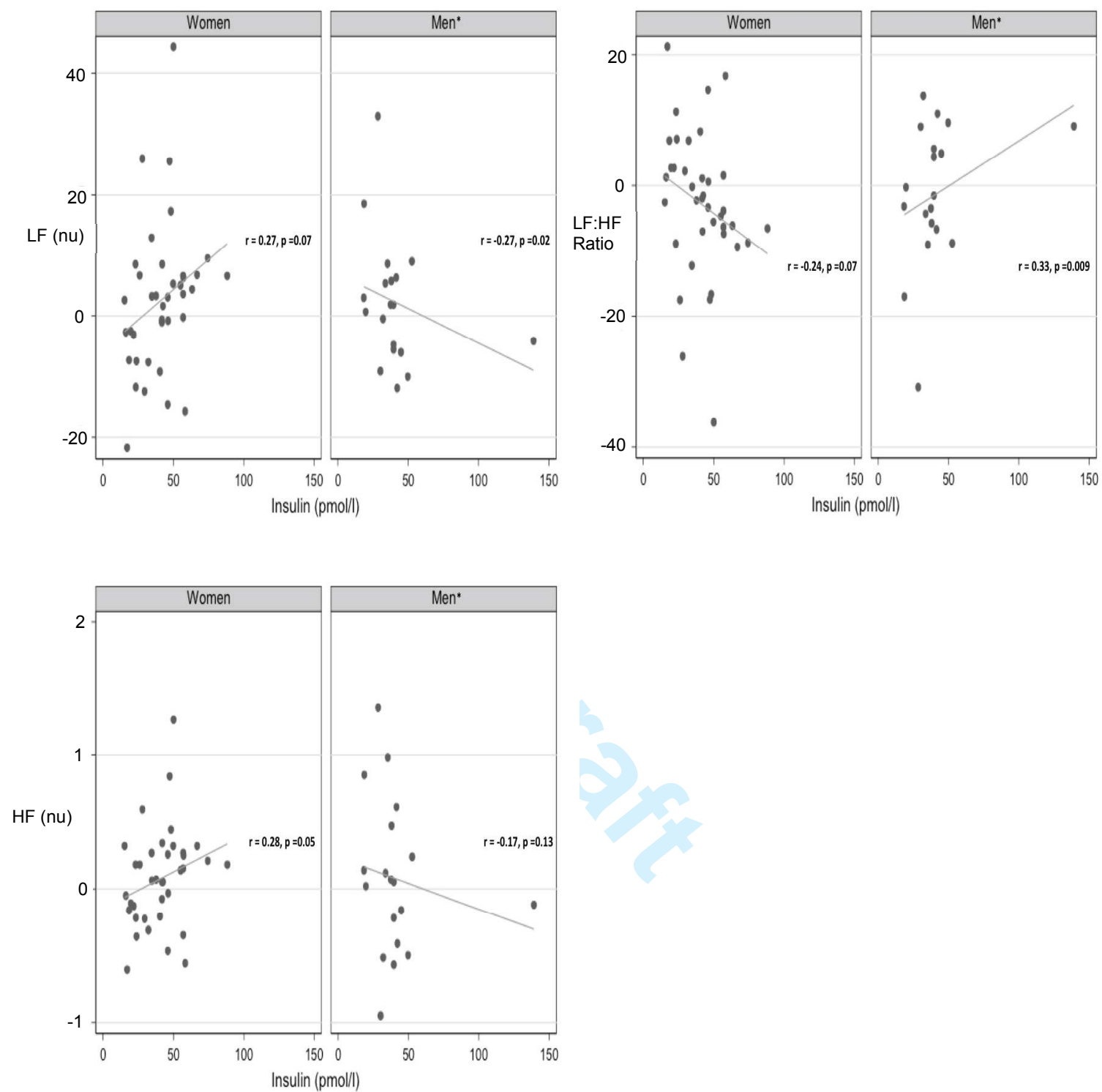

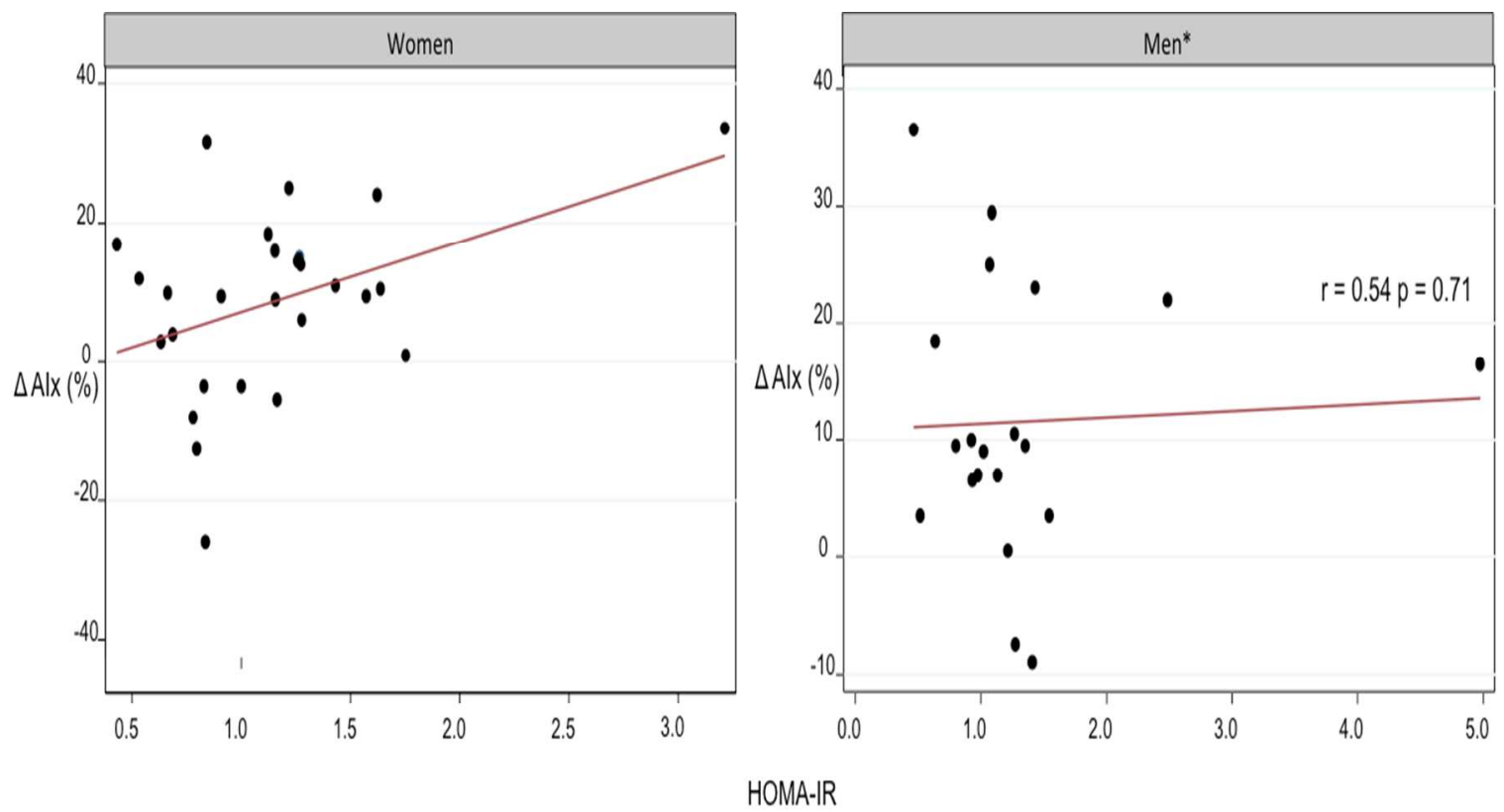

https://mc06.manuscriptcentral.com/cjpp-pubs 